\title{
Alpha-fetoprotein Epitope Peptide Promoted the Proliferation of Primary Hepatocellular Carcinoma Cells
}

\author{
Huanping Lin ${ }^{1}$, Xiaoping Wang ${ }^{1,{ }^{*}}$, Bing Xu ${ }^{1}$, Qian Zhao ${ }^{1}$, Ke-pei Zhang ${ }^{1}$, \\ Pengfei Zhang ${ }^{2}$ \\ ${ }^{1}$ Key Laboratory of Molecular Biology and Pathology, Shaanxi University of Chinese Medicine, \\ Xianyang, Shaanxi 712046, PR China \\ ${ }^{2}$ First Affiliated Hospital, Xi'an Jiaotong University, Xi'an, Shaanxi 710068, PR China \\ *Corresponding author, wxpphd72@yahoo.com
}

Keywords: Alpha-fetoprotein (AFP); Epitope Peptide; Proliferation; Hepatocellular Carcinoma (HCC)

Abstract. Alpha-fetoprotein (AFP) is an oncofetal protein during hepatocellular carcinoma (HCC) development which could promote tumor growth and inhibit active immunity, and it may serve as a target for immunotherapy. To further confirm the immunogenicity of AFP, we constructed a AFP eitope peptide vaccine which contained the specific antigen epitope. Our results demonstrated that although AFP epitope peptide induced a distinct level of antibody, it still inhibited dendritic cells, $\mathrm{CD}^{+} \mathrm{T}$ cells, natural killer cells responses and promoted the growth of AFP-expressing tumors. Our study suggests that AFP epitope peptide promoted the proliferation of hepatocellular carcinoma by inhibiting the effects of immune cells, which may be a better basis for further study on HCC immunotherapy.

\section{Introduction}

Hepatocellular carcinoma (HCC) is one of the most common malignancies over the world and accounts for as many as 1.2 million deaths annually.1 It is rising rapidly in China because of hepatitis $\mathrm{B}$ and $\mathrm{C}$ infections [1,2]. Although surgery and liver transplantation are the effective therapy, most patients still lose chance due to diagnosis at a late stage or underlying liver insufficiency in the setting of cirrhosis [3]. Novel therapies for HCC should be developed. A combined therapy is likely to prolong patients' life and living quality.

In the recent years, immunotherapy with therapeutic vaccination, which could manipulate and enhance the host's immune system to elicit antigen-specific responses to the tumors, has attracted attention widely. Much attention is attracted to tumor active immunity which purpose is to induce hosts' immune attack on tumor cells. 80\% of Hepatocellular carcinoma (HCC) have a high expressing rate of alpha-fetoprotein (AFP), which may serve as a target for immunotherapy [4-6]. AFP is an oncofetal protein during HCC development which could generate weaker and less reproducible antitumor protection [7]. Therefore, a vaccine specifically targeting AFP is particularly desirable.

In the present study, we investigated whether the immunogenicity of AFP peptide containing antigen epitope FMNKFIYEI, could be induced by way of intradermal immunization and further evaluated the immune responses induced by AFP peptide vaccine (AFP-P).

\section{Material and Methods}

Mice and Tumor Cell Lines. Six- to eight-week-old female BALB/C mice were purchased from the Experimental Animal Center at Fourth Military Medical University. All animals were maintained under specific-pathogen-free conditions, and all procedures were performed according to approved protocols and in accordance with recommendations for the proper care of laboratory animals. The investigation was approved by the Ethics Committee on animal Study at Shaanxi University of Chinese Medicine (2004-4B). mAFP-producing H22 mice hepatocellular carcinoma cells were 
kindly provided by College of Biomedicine, Xi’an Jiaotong University.

AFP Peptide Systhesis. Mouse AFP peptide containing epitope 158-166 amino acid, FMNKFIYEI, was synthesized from the ZiYuPeptides Co., Ltd (Shanghai, China). Lyophilized material was resuspended in sterile distilled water at $10 \mathrm{mg} / \mathrm{ml}$, aliquoted, and stored at $-70{ }^{\circ} \mathrm{C}$ until use. Protein concentration was determined using BCATM protein assay kit (Pierce Inc., Rockford, IL, USA). Aliquots of each conjugate were stored at $-70^{\circ} \mathrm{C}$ until use.

Mice Immunized with AFP-P Vaccine. Female BALB/C mice were randomly divided into AFP-P group, PBS group and empty group. Every group had 10 mice. Before injection, each group was diluted in saline to $100 \mu \mathrm{g} / 100 \mu \mathrm{l}$. Various groups of mice were injected into the left flank

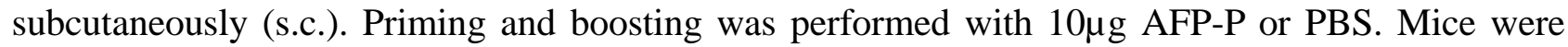
boosted s.c. with above proteins twice at 2 weeks intervals after the first priming. Two weeks after last immunization, splenocytes were harvested and diluted to different concentrations.

ELISA Assay. The ELISA was used to measure the antibody against AFP peptide and the cytokine IFN- $\gamma$ in splenocytes harvested from above immunized mice according to manufacturer's instruction. The OD values were obtained using an ELISA Reader System.

Granzyme B ELISA. $1 \times 10^{6}$ splenocytes harvested from above immunized mice were added to the 96 well microplate along with $5 \mu \mathrm{g} / \mathrm{ml}$ of AFP peptide containing $10 \%$ fetal bovine serum, 10 units/ml of mouse interleukin (IL)-2 (PEPRO Tech ET Ltd.). After cultured at $37{ }^{\circ} \mathrm{C}$ for $24 \mathrm{~h}$, granzyme B released by actived immune cells was measured using a mouse granzyme B ELISA kit (CSB-E08720m, Cusabio Biotech) according to manufacturer's instructions. Plates were counted on an ELISA reader at $490 \mathrm{~nm}$.

Cytotoxic T-lymphocyte (CTL) Assays. BALB/C mice were immunized subcutaneously (s.c.) as described above. Two weeks after the last boost, $2.5 \times 10^{7}$ splenocytes were cocultured with 5 $\mu \mathrm{g} / \mathrm{ml}$ of AFP peptide containing $10 \%$ fetal bovine serum, 10 units $/ \mathrm{ml}$ of mouse interleukin (IL)-2 in RPMI 1640 supplemented with $10 \% \mathrm{FCS}$ at $37{ }^{\circ} \mathrm{C}$ in $5 \% \mathrm{CO}_{2}$. After 5 days of stimulation, the viable splenocytes were recovered and used as effector cells, and the H22 cells or LLC cells were used as target cells. The Non-Radioactive Cytotoxicity Lactate Dehydrogenase (LDH) release assay Kit (Promega, 249 USA) was performed to measure the effector cells against H22 or LLC tumor cells in the ratios of 10:1, 20:1 and 40:1, according to the manufacturer's protocol. Specific lysis was calculated according to the formula: percent specific lysis $=[$ (experimental release value effector spontaneous release value - target spontaneous release value)/(target maximum release value - target spontaneous release value) $] \times 100$. Results shown are representative of experiments repeated three times.

Dendritic Cell, CD8 ${ }^{+}$T Cell and Natural Killer Cell Cytotoxicity Assays. To test dendritic cell, $\mathrm{CD}^{+} \mathrm{T}$ cell or natural killer cell response, a separate set of immunized mice were sacrificed and spleens were collected. Dendritic cells, $\mathrm{CD}^{+} \mathrm{T}$ cells or natural killer cells were purified and enriched from spleen suspensions with anti-CD $11^{+}$, anti-CD8 ${ }^{+}$or anti-NK Micro-Beads. The above-purified immune cells were cocultured with $5 \mu \mathrm{g} / \mathrm{ml}$ of AFP peptide containing $10 \%$ fetal bovine serum, 10 units/ml of mouse interleukin (IL)-2 in RPMI 1640 supplemented with 10\% FCS at $37{ }^{\circ} \mathrm{C}$ in $5 \% \mathrm{CO}_{2}$. After 5 days of stimulation, the splenocytes were used as effector cells and co-cultured with the H22 cells as described above.

In Vivo Tumor Therapeutic Experiments. To confirm whether AFP-P inhibited the growth of established tumors, BALB/C mice in every group were injected subcutaneously in the left flank with $5 \times 10^{5} \mathrm{H} 22$ cells per mouse on day 0 , and then injected subcutaneously in the right flank with different vaccination treatments on day 3, 10, and 17 as above described. H22 tumor cells were washed after enzymatic digestion and resuspended in $0.2 \mathrm{ml}$ of PBS per animal, then injected s.c. into the left flank, while PBS was used as control. The tumor growth was monitored every day. Tumor size was measured in two dimensions with calipers every 3 days one week after tumor inoculation. Tumor volume was calculated using the formula: $V=\left(a^{2} b\right) / 2$. Percentage of tumor-free mice was recorded and the survival of mice was monitored for eight weeks from the day of tumor challenge.

Statistical Analysis. All data expressed as means \pm S.D. Comparisons between individual data 
points were made using a Student's t-test. The frequencies of IFN- $\gamma$-producing splenic cells were valued using $\chi^{2}$ test. The Student's t test was performed to analyze the significance of differences between final tumor volumes of different groups of animals. $P<0.05$ was considered statistically significant.

\section{Results and Discussion}

AFP Peptide Vaccine Generate AFP-specific Antibody in Vivo. Two weeks after the last immunization, splenocytes were harvested and stimulated with AFP peptide. Immunization of BALB/C mice with AFP-P vaccine induced a significant rise of antibody compared with PBS control. About $126.45 \mathrm{ng} / \mathrm{ml}$ specific antibody for AFP peptide were detected per 1x10 ${ }^{6}$ splenocytes derived from the AFP-P vaccinated mice, compared with $54.62 \mathrm{ng} / \mathrm{ml}$ antibody derived from PBS vaccinated mice. Our data suggested that AFP-P vaccine elicited AFP-specific humoral response compared with the control group.

On the contrary, the concentration of IFN- $\gamma$ in the splenocytes from mice immunized with AFP-P were as similar as those from mice immunized with PBS (117.52 \pm 6.47 vs $114.38 \pm 8.75, \mathrm{P}>0.05)$. AFP-P immunized mice produced lower concentration of granzyme B than those immunized with PBS (59.48 \pm 6.59 vs $57.32 \pm 8.43, \mathrm{P}>0.05)$. The results indicated that AFP-P vaccine could not induce significant AFP-specific immune T cells response compared with the control group.

AFP-P Inhibit Potent AFP-specific DC Cell, $\mathrm{CD8}^{+} \mathrm{T}$ Cell and Natural Killer Cell Responses. Cytotoxicity assay showed that splenocytes cells from mice immunized with AFP-P exhibited lower cytolytic effects on H22 target cells than those from mice vaccinated with PBS (P < 0.01). In contrast, splenocytes from the PBS control showed a weaker ability of target cells lysis. Our results indicated that AFP peptide was an inhibitor for AFP specific immune cell activity.

To elucidate whether DC cells, $\mathrm{CD}^{+} \mathrm{T}$ cells or natural killer cells participate in the cytotoxic against $\mathrm{H} 22$ tumor cells, DC cell, $\mathrm{CD}^{+} \mathrm{T}$ cell and natural killer cell purified assay were determined. The purified and enriched DC cells, $\mathrm{CD}^{+} \mathrm{T}$ cells or natural killer cells from mice vaccinated with AFP-P showed lower cytolytic effects on $\mathrm{H} 22$ target cells. There was no significant difference from mice treated by AFP-P and PBS (P > 0.05). Our results indicated that the specific cytolytic activity against $\mathrm{H} 22$ tumor cells of DC cells, $\mathrm{CD}^{+} \mathrm{T}$ cells and natural killer cells were concurrently inhibited via AFP-P immunization.

AFP-P Vaccination Promote the Growth of H22 Tumors. The growth of tumor was monitored for about 4 weeks since day 7 post tumor inoculation. The mice receiving AFP-P or PBS developed tumor growth within 10 days after tumor challenge. Mice immunized with AFP-P demonstrated the higher average tumor volumes than mice immunized with PBS. There was a statistically significant difference in the growth promotion effect of H22 tumor between AFP-P and PBS vaccinated group (649.37 \pm 12.45 vs 346.75 $\pm 11.58, \mathrm{P}<0.01$ ). Different vaccinations greatly influenced the survival of mice. The mice immunized with AFP-P all died before day 30 and all the mice immunized with PBS still survived at day 35, whereas all the mice treated with PBS demised before day 42. These data suggested that AFP-P immunization could promote tumor growth and reduce the survival time of the tumor-bearing mice than PBS immunization alone, indicating that the vaccination of AFP-P induced no therapeutic effect against $\mathrm{H} 22$ tumor cells loaded in vivo. These results showed that vaccination with AFP-P could not eradicate previously inoculated AFP-expressing tumors in mice and inhibit the growth of tumors.

Recent studies on the immunodominant epitopes of AFP have provided a solution to the obstacle of HCC immunotherapy. AFP is produced at low serum levels after birth throughout life. The majority of human HCC overexpress the oncofetal antigen AFP, Mr 64,000 72,000 glycoprotein. Despite being exposed to high plasma levels of this oncofetal protein during embryonic development, body still displays a low immunity to it [8]. Butterfield has found that four peptides of human AFP processed and presented in the context of HLA-A0201, could be recognized by the human T cell repertoire, and could be used to generate AFP-specific CTL in human T cell cultures [9]. It was also found that murine immune system could generate T-cell responses to this oncofetal 
antigen. Therefore, it may be an ideal target for immunotherapy. But AFP immunization alone still results in lower levels of specific response and poorly reproducible protective immunity [4-7].

How to enhance host's active immunity to AFP may be an interesting strategy for HCC therapy. How to enhance host's active immunity to AFP may be an interesting strategy for HCC therapy. Previous studies on AFP specific immunothereapy for HCC included AFP plasmid immunization, AFP-transduced dendritic cells (DCs) immunization and AFP plasmid prime-AFP adenovirus boost immunization [10-14]. AFP plasmid immunization produced detectable but low levels of AFP specific $T$ cell responses and poorly reproducible protective immunity [4-7]. Additional enhancement of the T-cell stimulatory effect is DCs engineered to express murine AFP, which demonstrated a powerful ability to generate tumor-specific immune responses $[15,16]$. However, the need for costly cell culture procedures limited their wide availability for clinical use, and the unstable culture technique might yield tolerating vaccine [15]. AFP plasmid prime-AFP adenovirus boost immunization could engender significant AFP specific T-cell responses and protective immunity in mice [12,13]. But the miscellaneous procedures precluded their use. In the present study, we tested a novel strategy to induce antitumor immunity by a AFP epitope peptide vaccine. We found AFP peptide could elicit certain AFP-specific B-cell responses and produce a detectable level of anti-AFP antibody, compared with control group. Currently, the mechanism of antibody-dependent immune cell function is not well defined. We don't know whether humoral immunity mediated by B cells plays a primary role in anti-tumor effect, but we could reckon that anti-AFP antibody was likely to neutralize the AFP antigen or mediate the antibody-dependent immune cell cytotoxicity.

Protein-based vaccines have become an attractive approach to generate antigen-specific immunotherapy because of their simplicity, safety, efficacy and capacity for repeated administration. Protein vaccines generate effective cytotoxic T lymphocyte (CTL) and antibody responses by involvement of antigen presenting cells (APC) that stimulate $\mathrm{CD}^{+}$and $\mathrm{CD}^{+}{ }^{+} \mathrm{T}$ cells. Protein vaccines can be easily prepared on a large scale with high purity and stability and can also be repeatedly given to the same patient safely and effectively $[17,18]$. The features make protein vaccines a potentially attractive approach for cancer therapeutic vaccine development. This study showed that AFP peptide vaccine could inhibit the immunity of DC cell, CD8 ${ }^{+} \mathrm{T}$ cell and natural killer cell. Meanwhile, AFP-P promoted the development and growth of AFP-expressing tumor. Our study suggests that AFP epitope peptide promoted the proliferation of hepatocellular carcinoma by inhibiting the effects of immune cells, which lay a better basis for deep comprehend of the development of HCC and further study on HCC immunotherapy.

\section{Summary}

AFP peptide vaccine can generate an impressive humoral response although it inhibits the immunity of DC cell, $\mathrm{CD}^{+} \mathrm{T}$ cell and natural killer cel and promotes the proliferation of AFP-expressing tumors. The peptide vaccine containing tumor antigen epitope may be a promising approach for further study on cancer therapy.

\section{Acknowledgements}

This work is supported by Scientific Research Program Funded by Shaanxi Provincial Education Department (No.2007JK233, 14JS025), the Key Project of Ministry of Education of China (No.205002) and the National Natural Science Foundation of China (No.81172135, 81310108008).

\section{References}

[1] D.F. Schafer, M.F. Sorrell, Hepatocellular carcinoma, Lancet, 353 (1993) 1253-1257.

[2] Z.Y. Tang, Hepatocellular carcinoma--cause, treatment and metastasis, World J. Gastroenterol. 7 (2001) 445-454. 
[3] R. Wong, C. Frenette, Updates in the management of hepatocellular carcinoma, Gastroenterol. Hepatol. (NY). 7 (2011) 16-24.

[4] P. Hanke, C. Rabe, M. Serwe, S. Böhm, C. Pagenstecher, T. Sauerbruch, W.H. Caselmann, Cirrhotic patients with or without hepatocellular carcinoma harbour AFP-specific T-lymphocytes that can be activated in vitro by human alpha-fetoprotein, Scand. J. Gastroenterol. 37 (2002) 949-955.

[5] J.A. Marrero, K.S. Henley, The role of serum biomarkers in hepatocellular carcinoma surveillance, Gastroenterol. Hepatol. (N Y). 7 (2011) 821-823.

[6] J.H. Zhong, H. Li, L.Q. Li, X.M. You, Y. Zhang, Y.N. Zhao, J.Y. Liu, B.D. Xiang, G.B. Wu, Adjuvant therapy options following curative treatment of hepatocellular carcinoma: a systematic review of randomized trials, Eur. J .Surg. Oncol. 38 (2012) 286-295.

[7] A. Saeki, K. Nakao, Y. Nagayama, K. Yanag, K. Matsumoto, T. Hayashi, H. Ishikawa, K. Hamasaki, N. Ishii, K. Eguchi, Diverse efficacy of vaccination therapy using the alpha-fetoprotein gene against mouse hepatocellular carcinoma, Int. J. Mol. Med. 13 (2004) 111-116.

[8] H.F. Deutsch, Chemistry and biology of a-fetoprotein, Adv. Cancer Res. 56 (1991) 253-312.

[9] L.H. Butterfield, A. Koh, W. Meng, C.M. Vollmer, A. Ribas, V. Dissette, E. Lee, J.A. Glaspy, W.H. McBride, J.S. Economou. Generation of human T-cell responses to an HLA-A2.1-restricted peptide epitope derived from alpha-fetoprotein, Cancer Res. 59 (1999) 3134-3142.

[10] W.S. Meng, L.H. Butterfield, A. Ribas, J.B. Heller, V.B. Dissette, J.A. Glaspy, W.H. McBride, J.S. Economou, Fine specificity analysis of an HLA-A2.1-restricted immunodominant $\mathrm{T}$ cell epitope derived from human alpha-fetoprotein, Mol. Immunol. 37 (2000) 943-950.

[11] C.F. Grimm, D. Ortmann, L. Mohr, S. Michalak, T.U. Krohne, S. Meckel, S. Eisele, J. Encke, H.E. Blum, M. Geissler, Mouse alpha-fetoprotein-specific DNA-based immunotherapy of hepatocellular carcinoma leads to tumor regression in mice, Gastroenterology, 119 (2000) 1104-1112.

[12] P. Hanke, M. Serwe, F. Dombrowski, T. Sauerbruch, W.H. Caselmann, DNA vaccination with AFP-encoding plasmid DNA prevents growth of subcutaneous AFP-expressing tumors and does not interfere with liver regeneration in mice, Cancer Gene. Ther. 9 (2002) 346-355.

[13] X.P. Wang, G.Z. Liu, A.L. Song, H.Y. Li, Y. Liu, Antitumor immunityinduced by DNA vaccine encoding alpha-fetoprotein/heat shock protein 70, World J. Gastroenterol. 10 (2004) 3197-3200.

[14] W.S. Meng, L.H. Butterfield, A. Ribas, V.B. Dissette, J.B. Heller, G.A. Miranda, J.A. Glaspy, W.H. McBride, J.S. Economou, alpha-Fetoprotein-specific tumor immunity induced by plasmid prime-adenovirus boost genetic vaccination, Cancer Res. 61 (2001) 8782-8786.

[15] J. Banchereau, R.M. Steinman, Dendritic cells and the control of immunity, Nature, 92 (1998) 245-252.

[16] C.M. Jr Vollmer, F.C. Eilber, L.H. Butterfield, A. Ribas, V.B. Dissette, A. Koh, L.D. Montejo, M.C. Lee, K.J. Andrews, W.H. McBride, J.A. Glaspy, J.S. Economou, Alpha-fetoprotein-specific genetic immunotherapy for hepatocellular carcinoma, Cancer Res. 59 (1999) 3064-3067.

[17] J.J. Donnelly, J.B. Ulmer, J.W. Shiver, M.A. Liu, DNA vaccines, Annu. Rev. Immunol. 15 (1997) 617-648.

[18] S. Gurunathan, D.M. Klinman, R.A. Seder, DNA vaccines: immunology, application, and optimization, Annu. Rev. Immunol. 18 (2000) 927-974. 